\title{
An internal examination training system supporting abnormal labor conditions
}

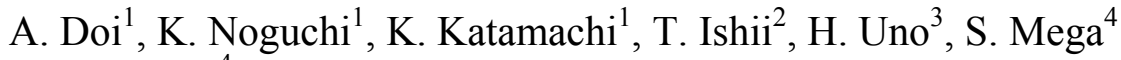 \\ \& K. Matsui ${ }^{4}$ \\ ${ }^{1}$ Iwate Prefectural University, Japan \\ ${ }^{2}$ The Japanese Red Cross Hokkaido College of Nursing, Japan \\ ${ }^{3}$ KOKEN Co., Ltd, Japan \\ ${ }^{4} \mathrm{JFP}$, Inc., Japan
}

\begin{abstract}
For obstetricians and midwives, "internal examination" refers to an important diagnostic technique in which the progress of labor is examined using the index and middle fingers inserted into the vagina or rectum. Training of this internal examination technique has been commonly performed using a model of the human body (manikin). However, with this method, it was impossible to determine visually where and how the examining fingers are touching, making it difficult for trainers to teach advanced examination skills efficiently and evaluate training achievements. Against this background, we have developed a training system for internal examination that enables simulation of normal and abnormal conditions of labor by detecting the position and direction of the examining fingers in real-time via tactile and visual perceptions using anatomical and virtual models. This system allows trainees to experience both normal and abnormal fetal descent into the pelvis.
\end{abstract}

Keywords: virtual models, manikin, magnetic sensor, internal examination, a training system.

\section{Introduction}

Previously developed training systems for internal examination include our own system [1-3], ePelvis [4-6] developed by a group from Stanford University, and the peripartum diagnosis/delivery assistance training system [7]. The ePelvis attaches several sensors inside the mother's body and is not suitable for close 
monitoring of fingers and the evaluation of examination techniques. The peripartum diagnosis/delivery assistance training system is basically a visual learning system using video images, and is suitable for teaching and explanation but not for training of the internal examination itself. Our approach in our training systems described in references [1-3] is different in comparison with the two systems, ePelvis and the peripartum diagnosis/delivery assistance training system. We utilize magnetic sensors that are attached with two fingers, and monitor the motions of the two fingers during an internal examination.

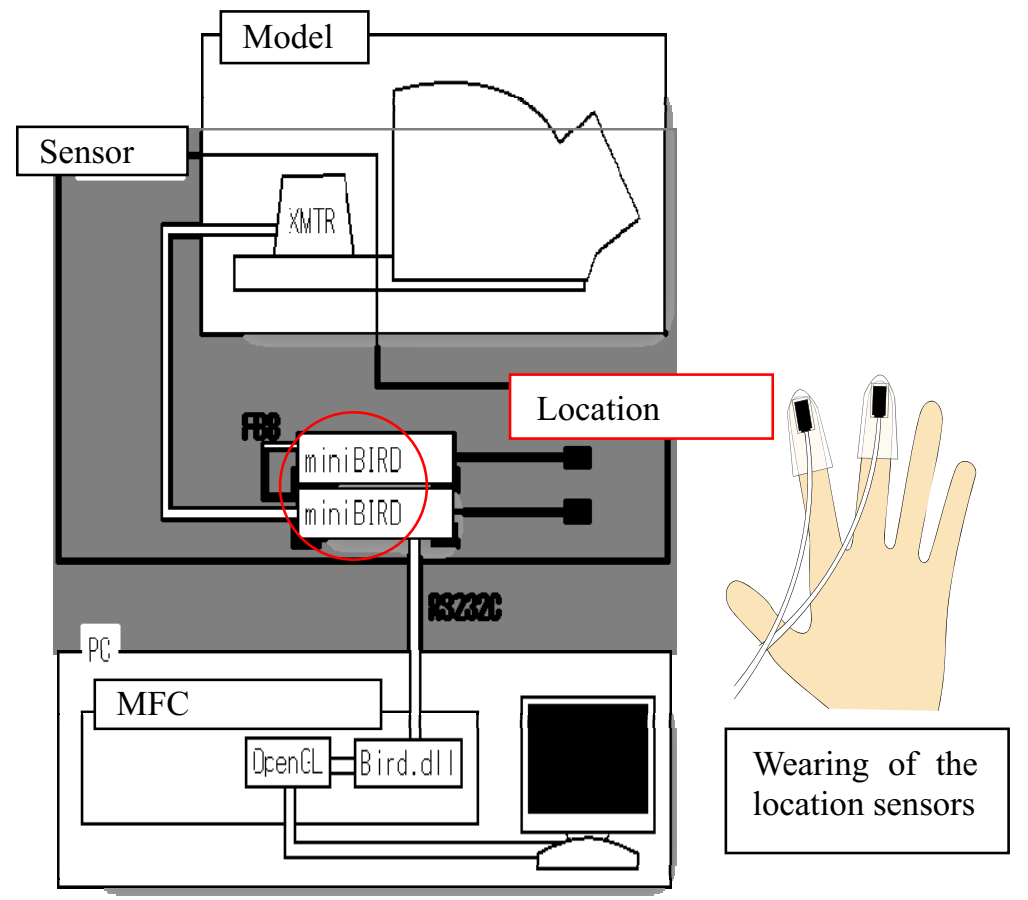

Figure 1: $\quad$ System configuration.

Our previous system was used to simulate the normal labor condition and thus was not suitable for simulating various abnormalities that can occur during labor. We thus developed a system based on fetal models that could reproduce various abnormal labor conditions (frank breech presentation, complete breech presentation, placenta previa, and face presentation). Our newly developed internal examination training system consists of models of maternal body parts, including the vagina and the uterine ostium, and fetal body parts (anatomical models), a personal computer (PC), and a magnetic sensor (Fig. 1). The magnetic sensor consists of a transmitter (XMTR), a location sensor, and a controller (miniBIRD, Ascension Technology Corp.). Magnetic information transmitted from the transmitter is detected by the location sensor, and the location information is received by the PC. The location sensor is attached and 
fixed to a fingerstall made of silicon rubber, which is worn on the index and middle fingers, with which the internal examination is performed.

\section{System overview}

Figure 2 shows the external appearance of the training system. The system supports normal labor conditions of one-finger dilatation, two-finger dilatation, full dilatation and type $\mathrm{C}$ (another type of full dilatation) and abnormal labor conditions of frank breech presentation, complete breech presentation, placenta previa and face presentation. Other conditions can be added if necessary. The monitor displays geometric models (i.e. virtual models of the human body, examining fingers, etc.) of the same size as the anatomical models. An advantage of the system is that it can display on the monitor the position and the direction of the examining fingers during an internal examination via the magnetic sensors worn on the examining fingers. The system thus allows trainers to evaluate visually the skill level and accuracy of examination techniques on the monitor. Inside the human body model shown in Figure 2 is a guiding structure to install models of the pelvis and the fetus. An appropriate anatomical model of the fetus should be placed in the human body model before starting training. The skin in the virtual model was created by measuring the skin with a non-contact 3D digitizer, and converting this to a polygonal shape.
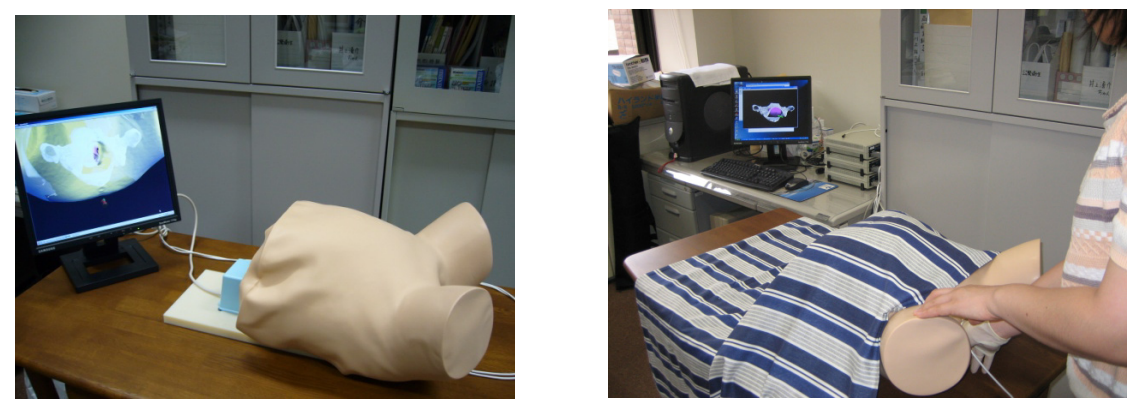

Figure 2: Our internal examination training system.

The entire fetal models are created by a computer aided design (CAD) software. All generated polygonal models are combined, and both color and optical information are added. The anatomical models of the pelvis and the body were created as follows: a 3D image of the pelvis was taken with a 3D-computed tomography (CT) system, the 3D image was appropriately smoothed and isosurfaced, and the surface shape of the pelvis and the body was created. The anatomical model of the pelvis and the fetus was made by a rapid proto-typing device (3D printer). The skin of the mother's body enclosing the pelvis was created by plastic material and vinyl cloth. 


\section{Our training method}

Eight anatomical models of the fetus, including four normal and four abnormal models, were prepared. Figure 3 shows four normal cases (left top: type $\mathrm{C}$ (near full dilation), right top: full dilation, left bottom: two-finger dilation, right bottom: one-finger dilation). Figure 4 shows four abnormal cases (left top: incomplete foot presentation, right top: complete breech presentation, left bottom: placenta previa, right bottom: face presentation).

These models create only the parts that are touched by the examining fingers, and are displayed together with the whole image of the fetus. After a fetal model was placed in the system, the virtual model of the selected fetal model was selected on the dialogue on the display, and the anatomical model and the virtual model were adjusted. The position of both anatomical and virtual models is adjusted by using the location sensor.

Figure 5 shows how a fetal model is displayed when it has been changed (before and after change). Figure 6 shows the dialogue for the selection of an anatomical model of a fetus (normal one-finger dilatation has been selected) and the control displays of each model (i.e. models of the mother's skin, pelvis, vagina, and fingers). The display control allows the selection of the constitutive models to display, the mode of display (i.e. wireframe (display with lines) or shading), transparency, and the display color. The system can also display internal views with four cross-sectional images (left, right, front, and back views) (Fig. 7).

Normal and abnormal conditions of different fetuses can be incorporated into the system by performing the following actions: 1) creating an anatomical model of the fetus; 2) measuring or modeling the anatomical model to create a virtual model; and 3) registering the new anatomical and virtual models into the system. The positions of the anatomical and virtual models can be adjusted automatically simply by indicating the zero point of a scale called "station" with an index finger pointer on the screen at the first application start-up.

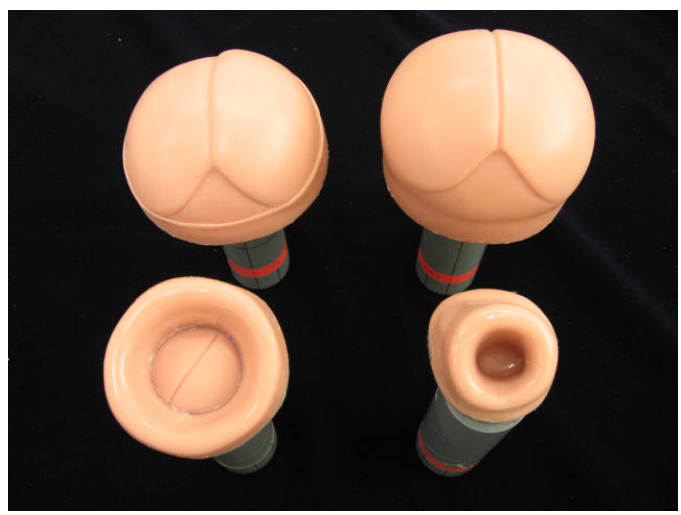

Figure 3: $\quad$ Anatomical models of fetus for normal cases. 


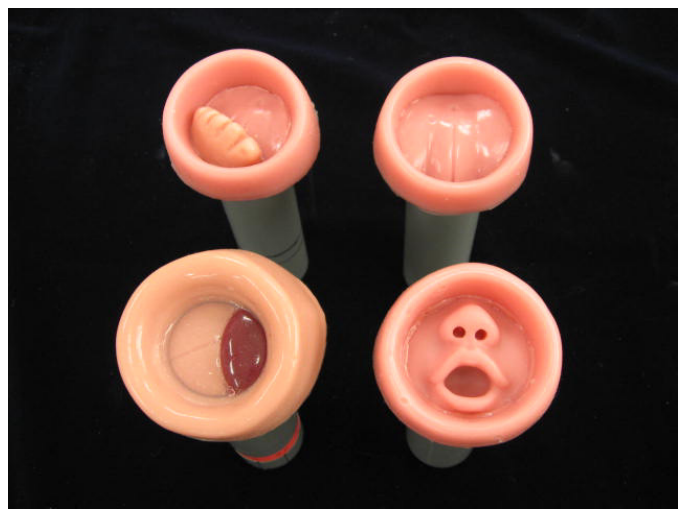

Figure 4: Anatomical models of fetus for abnormal cases.
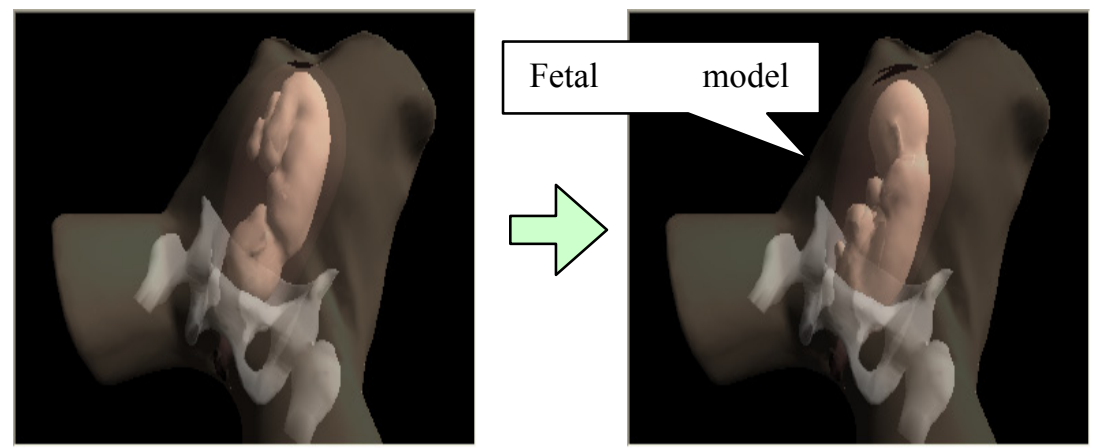

Figure 5: Change of a fetal model.

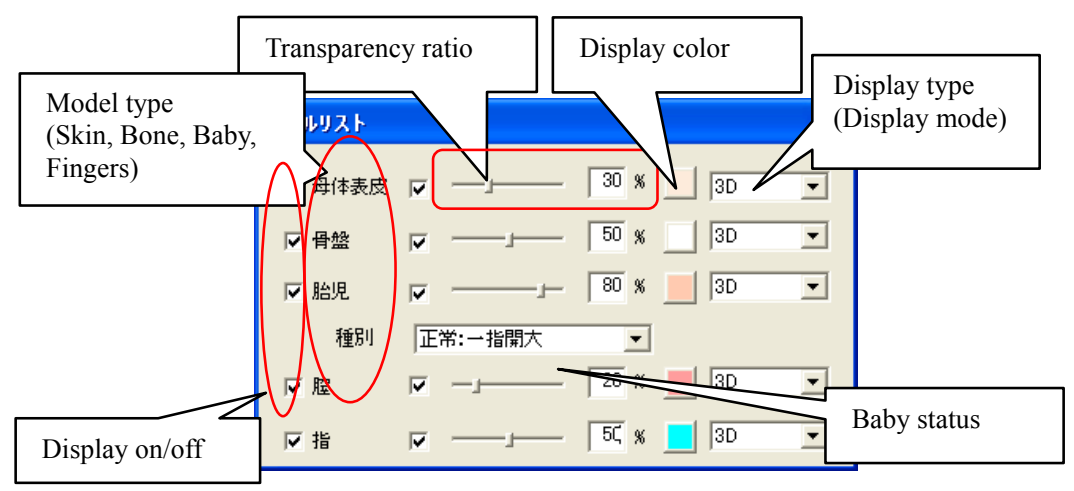

Figure 6: Fetal model selection dialogue and display function. 


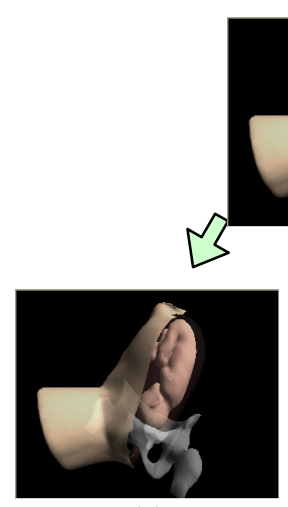

(a)

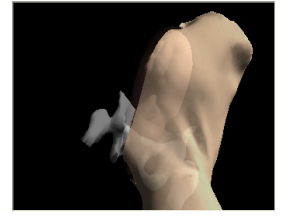

(b)

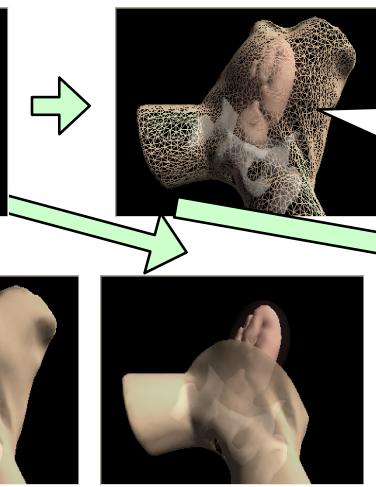

(c)
Skin of the mother displayed in wireframe model

Figure 7: Display function of the internal examination training system. (a) Cross-section: left view. (b) Cross-section: right view. (c) Crosssection: anterior view. (d) Cross-section: posterior view.

\section{Fetal presentation}

Fetal presentation refers to the orientation of the fetus in the uterus and is classified as "longitudinal presentation", "transverse presentation," or "oblique presentation". Longitudinal presentation is further divided based on the position of the fetal head into the "head presentation" (i.e. the fetal head is facing down) and "pelvic presentation" (i.e. the fetal pelvis is facing down; also known as the breech position).

All fetal presentations other than head position are considered abnormal. It is known that less than $5 \%$ of fetuses are delivered in breech position. Delivery of fetuses in breech position carries a higher risk of experiencing difficulty in pushing out the head and causing compression of the umbilical cord than does delivery of fetuses with their head facing down (i.e. head presentation).

Breech position is roughly divided into frank breech presentation, full breech presentation, knee presentation, and foot presentation. The fetal presentation in which fetal feet are facing up and the hips facing down is referred to as the frank breech presentation, that in which both feet are facing down is referred to as complete breech presentation, and that in which only one foot is facing up is referred to as incomplete breech presentation.

"Knee presentation" is the condition in which the fetal knee is flexed and facing down during delivery (i.e. the fetus descends with its knee presenting first), and is further divided into "complete knee presentation" (i.e. both knees facing down) and "incomplete knee presentation" (i.e. only one knee facing down). "Foot presentation" is the condition in which the fetus descends with its extended legs presenting first during delivery. Some breech babies can be delivered naturally through the vaginal canal if they are in frank breech presentation or full breech presentation. Breech babies in knee or foot 
presentation have less chance to be delivered naturally and are usually delivered by Caesarian section (cutting) [8].

\section{System evaluation}

We conduct a survey of eight students at our university, and make inquiries for our system, and evaluate it for understanding where the position of ischiadic thorn, baby head, and uterine ostium. We also check that it is easy to understand the rotation of baby head and the open of the uterine ostium. In the lecture of an internal examination, first we teach an internal examination in the classical method using a manikin only. Next, we teach them by using our system and make inquiries for our system with unregistered style. We check understanding of the students for several questions before and after looking images in our system. The result of several questions shows that the understanding of all students is improved by using our system [9].

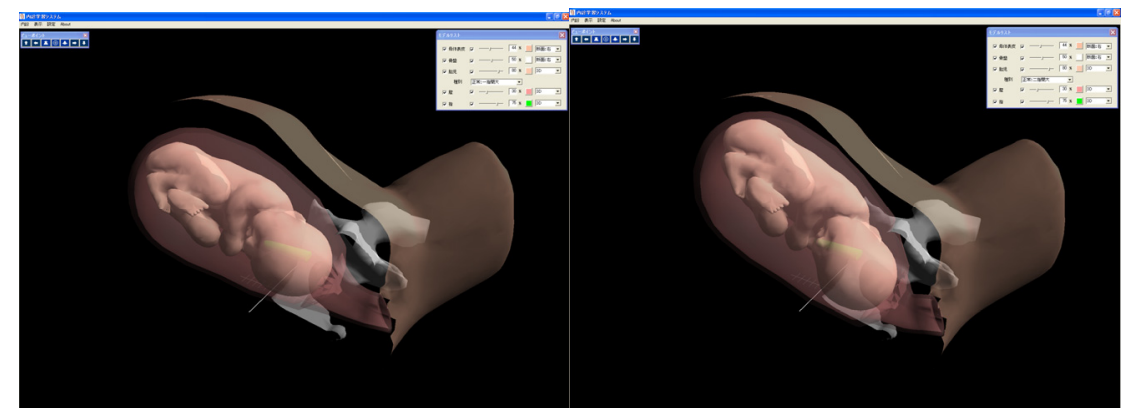

Figure 8: Fetal models of normal conditions (left: one-finger dilatation; right: two-finger dilatation).

Figures 8 and 9 show fetal models of normal conditions supported by the internal examination training system (i.e. one-finger dilatation, two-finger dilatation, type $\mathrm{C}$ (almost full dilatation), and full dilatation). Figures 10 and 11 show fetal models of abnormal conditions (incomplete foot presentation (frank breech presentation), complete breech presentation, placenta previa, and face presentation). We have been evaluating the usefulness of the present technique by disseminating its use as a learning tool and presenting it at international meetings and exhibitions. At the exhibition of the $21^{\text {st }}$ Japan Academy of Midwifery Scientific Meeting (Oita prefecture in Japan, March 10 and 11, 2007), we presented and demonstrated our internal examination training system and a number of people experienced the training procedure and answered a questionnaire about the usefulness of the system. Over 100 people attended our presentation, approximately 60 of whom experienced the training procedure and provided feedback.

Most of those who experienced the training system at the exhibition of the $21^{\text {st }}$ Japan Academy of Midwifery Scientific Meeting provided positive 
comments, although some requested improvements or the addition of functions. These requests included the addition of various abnormal labor conditions, more measurement functions (e.g. distance between two fingers and distance to the head of fetus), an automatic calibration function, and an examination rating function.

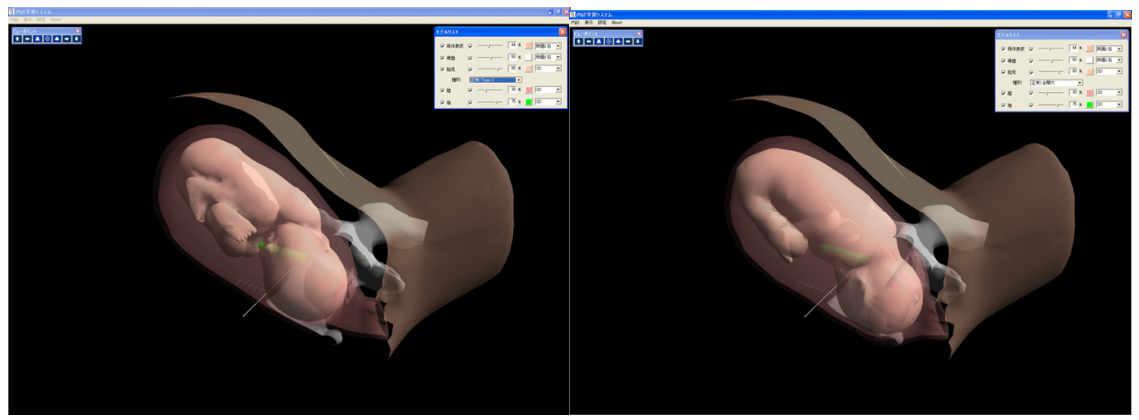

Figure 9: Fetal models of normal conditions (left: type C; right: full dilatation).

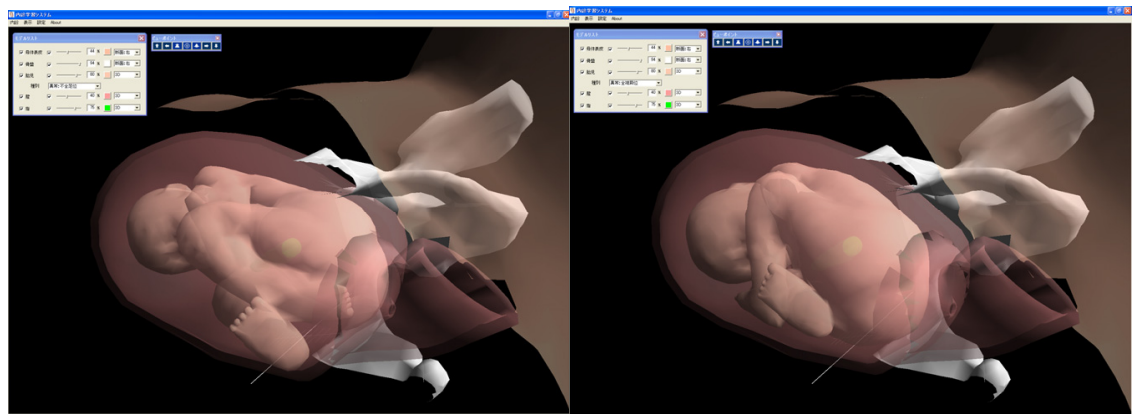

Figure 10: Fetal models of abnormal conditions (left: incomplete foot presentation; right: complete breech presentation).

We have satisfied one of the requests by developing 4 abnormal labor conditions (frank breech presentation, complete breech presentation, placenta previa and face presentation) and have included them in the latest training system. We have also added measurement functions using one or two fingers; the location sensors mounted on the index and middle fingers enable the acquisition of position information in space (i.e. spatial position and rotation). Using this measurement function, we can precisely measure spatial distances estimated on the basis of the distance between the two fingers or the measurer's experience. In future studies, we aim to perform more detailed examinations of abnormal labor conditions. 


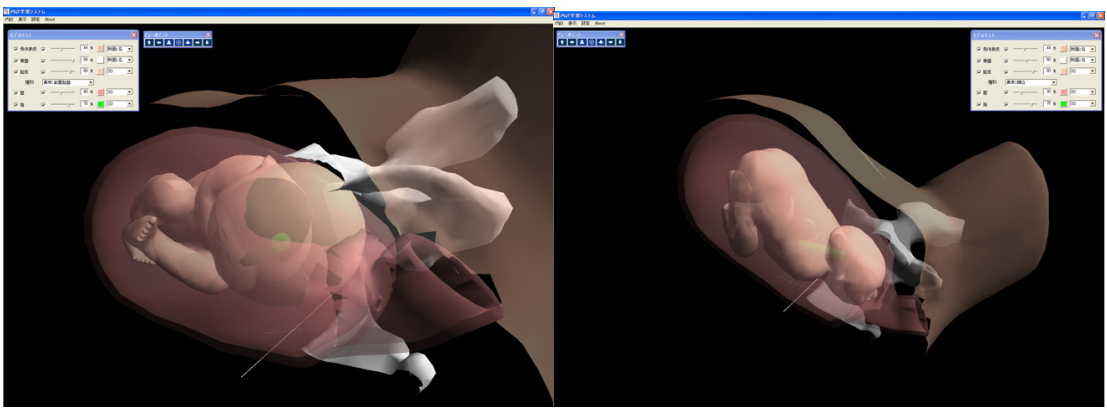

Figure 11: Fetal models of abnormal conditions (left: placenta previa; right: face presentation).

\section{Conclusion}

We have developed an internal examination training system that supports the learning of abnormal labor conditions. The system provides a unique learning experience that cannot be gained in daily practice. The use of the system in teaching practice will help to nurture competent obstetricians and midwives in a short period.

On the basis of specialist feedback, we included 4 abnormal fetal models in the training system. The availability of additional abnormal fetal models will make the system more relevant to actual clinical situations. Although the present system was developed specifically for internal examination of the fetus, it can also be applied for such purposes as medical training and preoperative planning for other parts of the body (e.g. thorax and abdomen) by using different anatomical and virtual models.

\section{References}

[1] Ishii, T., Doi, A., Katamachi, K., Noguchi, K., and Uno, H., Medical Training Device, Japan Patent Application No. 2005-032614, Reference No. 4-1130, Receipt No. 50500225450, Patent Applicant: KOKEN Co., Ltd. (August 2004)

[2] Ishii, T., Doi, A., Katamachi, K., Noguchi, K., and Uno, H., Medical Training Device, International patent application (European Patent Office), Reference No. GP05-1033PCT, Receipt No. 50600235406, Application No. Notification: PCT/JP2006/30219, Patent Applicant: KOKEN Co., Ltd.

[3] Doi, A., Matsui, K., Katamachi, K., Noguchi, K., Ishii, T., Uno, H., A computer assisted medical training system for checking status of delivery by using virtual reality technique and physical models, CARS2007, p. 156, 2007.

[4] Carla M. Pugh, et al., The E-Pelvis: A Pelvic Examination Simulator, SUMMIT (Stanford University Medical Media \& Information 
Technologies) Project Overview, http://summit.stanford.edu/publications/ tear_index.html

[5] SUMMIT (Stanford University Medical Media \& Information Technologies, e-Pelvis, http://summit.stanford.edu/research/epelvis.html

[6] Shreve, J., ePelvis for simulation of gynecologic internal examination, http://hotwired.goo.ne.jp/news/technology/story/20020320307.html

[7] MC Medica Shuppan, 3D CG Peripartum Diagnosis/Delivery Assistance Training System, http://www.medica.co.jp/3d-bunben/\#top

[8] Yajima, S., Nakano, H., and Taketani, Y., NEW Gynecologic Sciences, Nankodo; $2^{\text {nd }}$ Edition (July 2004).

[9] Noguchi, K., and Ishii, T., Computer-assisted Educational Evaluation, Presentation at Japan Academy of Nursing Education, August 2, 2006. 